\title{
Blurred Image Detection and Classification
}

\author{
Ping Hsu and Bing-Yu Chen \\ National Taiwan University \\ vivace@cmlab.csie.ntu.edu.tw; robin@ntu.edu.tw
}

\begin{abstract}
Digital photos are massively produced while digital cameras are becoming popular, however, not every photo has good quality. Blur is one of the conventional image quality degradation which is caused by various factors. In this paper, we propose a scheme to detect blurred images and classify them into several different categories. The blur detector uses support vector machines to estimate the blur extent of an image. The blurred images are further classified into either locally or globally blurred images. For globally blurred images, we estimate their point spread functions and classify them into camera shake or out of focus images. For locally blurred images, we find the blurred regions using a segmentation method, and the point spread function estimation on the blurred region can sort out the images with depth of field or moving object. The blur detection and classification processes are fully automatic and can help users to filter out blurred images before importing the photos into their digital photo albums.
\end{abstract}

\section{Introduction}

Image degradation comes into existence in different environments: unstable camera, night scene, moving object, etc. Many users take photos excursively and it turns out that there exist a lot of low quality photos. It is not convenient that the users have to take much time to find these defective photos out after a tour. The goal of blur classification in this paper is to help the users to automatically detect the blurred photos and classify them into some categories to let the users make further decisions.

Since the image gradient model is highly related to image blurring [1], our blur detector uses support vector machine (SVM) to verify the gradient model and estimate the magnitude of blur in an image, and then the blurred image is also determined to globally or locally blurred. Either camera shake or out of focus may cause globally blurred image, yet we can verify them by the point spread function (PSF). The PSF describes the response of an image to a point source or point object. After calculating the PSF of an image, the globally blurred image can be classified into camera shake or out of focus. Simultaneously the locally blurred image can be classified into two types: depth of field and moving object. We find the blurred regions by segmentation method, and the PSF estimation on the blurred region can sort out the image with depth of field or moving object. 
Therefore, every image, by our blur detector, can be classified into one of the following five types: non-blurred, camera shake, out of focus, moving object, and depth of field. Fig. 1 shows the procedure of the blur detection.
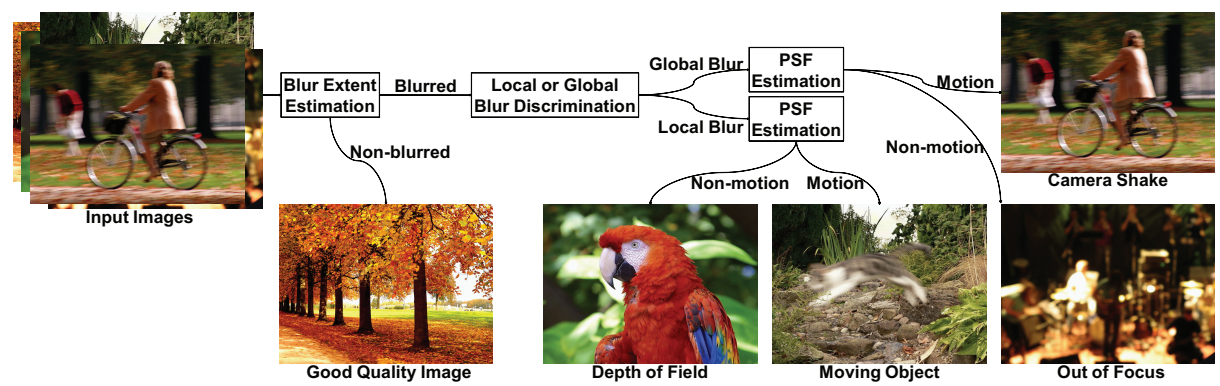

Fig. 1. The procedure of the blur detection.

\section{Related Work}

Tong et al. [2] proposed a scheme that makes the use of the ability of Harr wavelet transform in both discriminating different types of edges and recovering sharpness from the blurred version, and then determines whether an image is blurred or not and to what extent an image is blurred. Elder et al. [3] proposed an algorithm to detect local scale control and localize the edges over a broad range of the blur scale. This method for edge detection leads to a method for estimating the local blur of image contours.

Ben-Ezra et al. [4] proposed a hybrid image system that uses a secondary detector to estimate the motion in the image. Combining the blurred image and the acquired motion information, a PSF is estimated that represents the path of the camera during the integration. Rooms et al. [5] assumed the PSF can be modeled with a single parameter, and they used a Gaussian function to estimate the single-parameter PSF from a single observation of a degraded image. Fergus et al. [1] adopted a variational Bayesian approach to estimate the PSF of an image. Given the grayscale blurred image $B$, the blur kernel $K$ and the latent image $L$ are going to be estimated by finding the values with the highest probability from the statistics of $L$. These statistics are based on the image gradients rather than the intensities, so the optimization is working on gradient domain. The variational algorithm minimizes a cost function that can represent the distance between the approximation and the true posterior. The updates are repeated until the change converges. The mean of the distribution is then taken as the final value of $K$.

Pedro et al. [6] proposed an algorithm using a graph-based representation of the image and applied it to image segmentation. Let $G=(V, E)$ be an undirected graph with vertices $v_{i} \in V$ to be segmented and edges $\left(v_{i}, v_{j}\right) \in E$ corresponding 
to pairs of neighboring vertices. A segmentation $S$ is a partition of $V$ such that each component $C \in S$ is a connected component in graph $G^{\prime}=\left(V, E^{\prime}\right)$ where $E^{\prime} \subseteq E$. Let $S^{0}$ be the initial segmentation, where each vertex $v_{i}$ is in its own component, and the algorithm merges the vertices repeatedly. After $m$ steps, $S^{m}$ is the output that segments $V$ into $r$ components $S=\left(C_{1}, \ldots, C_{r}\right)$.

\section{Blur Extent Estimation}

\subsection{Image Gradient Model}

Gradient can be considered as a gradation from low to high values. The gradient of an image is a function that at each pixel the gradient vector points in the direction of largest possible intensity increase with the components given by the derivatives in the horizontal and vertical directions. The gradient of a two variables function $F(x, y)$ is defined as

$$
\nabla F=\frac{\partial F}{\partial x} \hat{i}+\frac{\partial F}{\partial y} \hat{j}
$$

If we define $F$ as the source image, $G_{x}=\frac{\partial F}{\partial x} \hat{i}$ and $G_{y}=\frac{\partial F}{\partial y} \hat{j}$ are two images which at each pixel contain the horizontal and vertical derivative values. At each pixel in the image, the resulting gradient values can be combined to give the

gradient magnitude, using $G=\sqrt{G_{x}^{2}+G_{y}^{2}}$. We can also calculate the gradient's direction as $\theta=\arctan \left(G_{y} / G_{x}\right)$.

Fig. 2 shows that the gradient histogram distribution of a clear image is massive on small values but there also exist some small noises on large values. Besides the magnitude histogram, we can find that every direction has almost the same probability in the direction histogram. On the contrary, the gradient magnitude distribution of a blurred image is almost empty on the large values and there only exists small values, and the gradient direction histogram also shows there are some values higher than others. According to the difference of the gradient distribution between a normal image and a blurred image, we can discriminate them by examining an image's gradient histogram.

\subsection{Support Vector Machines}

SVM, which has been proposed as a very effective method for pattern recognition $[7,8]$, is a set of related supervised learning methods used for classification. A special property of SVM is that it minimizes the classification error and maximizes the geometric margin. SVM maps input vectors to a higher dimensional space and constructs a hyperplane to separate the data into two groups. Two parallel hyperplanes are built up on each side of the hyperplane that maximize the distance between the two groups.

Given a training set of pre-labeled data points $\left(x_{i}, y_{i}\right), i=1, \ldots, l$ where $x_{i} \in R^{n}$ belongs to either of two classes and $y_{i} \in\{1,-1\}$ denotes the label, the 

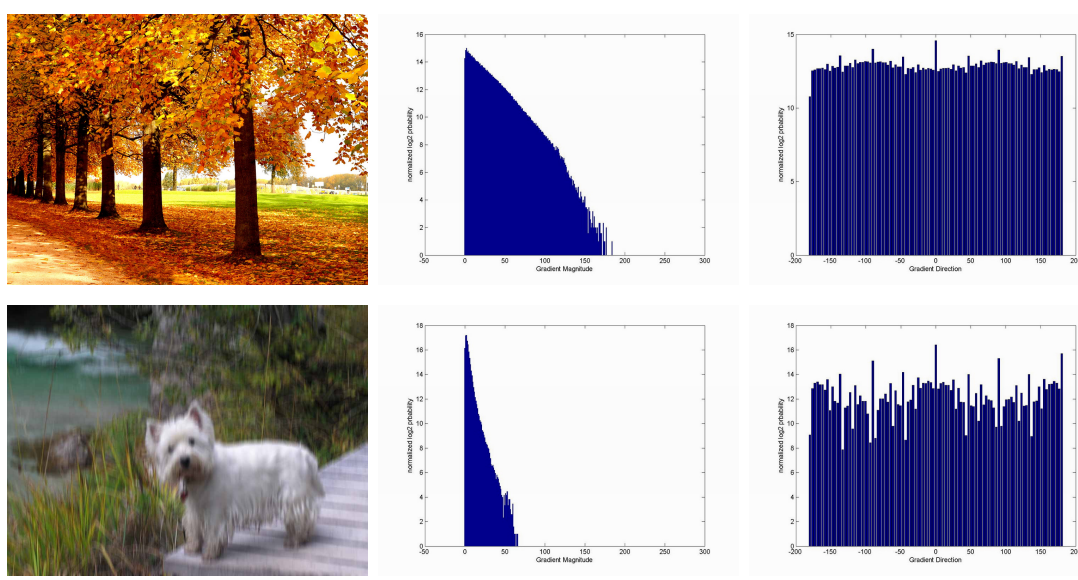

Fig. 2. Left: original images. Middle: gradient magnitude histogram. Right: gradient direction histogram.

support vector machines, to establish a hyperplane that can divide all $x$ with maximizing the distance between the two classes, solves the following optimization problem:

$$
\begin{array}{lr}
\text { Minimize } & \frac{1}{2} \mathbf{w} \cdot \mathbf{w}+C \sum_{i=1}^{N} \xi_{i} \\
\text { Subject to } & y_{i}\left(\mathbf{w} \cdot \phi\left(x_{i}\right)+b\right) \geq 1-\xi_{i}, \quad \xi_{i}>0
\end{array}
$$

The nonnegative variable $\xi=\left\{\xi_{1}, \xi_{2}, \ldots \xi_{N}\right\}$ allows a small number of misclassified points for better generalization, and $C>0$ is the penalty parameter of the error term. Furthermore, $K\left(x_{i}, x_{j}\right)=\Phi\left(x_{i}\right) \cdot \Phi\left(x_{j}\right)$ is called the kernel function and we use the radial basis function $(\mathrm{RBF})$ as the kernel function where $K\left(x_{i}, x_{j}\right)=e^{-\left|x_{i}-x_{j}\right|^{2} / 2 \sigma^{2}}$.

Instead of predicting the label, sometimes we are looking for a probability prediction $\operatorname{Pr}(y=1 \mid x)$, Platt [9] proposed a method to approximate the posterior by a sigmoid function:

$$
\operatorname{Pr}(y=1 \mid x) \approx P_{A, B}(x) \equiv \frac{1}{1+\exp (A f(x)+B)},
$$

where the parameters $(A, B)$ are estimated by solving a maximum likelihood problem.

\subsection{Blur Extent Estimation Using SVM}

There are two types of training data: clear images and blurred images. Every labeled image $I_{i}, i=1 \ldots l$ is converted to grayscale, so the gradient magnitude 
and direction maps can be built up first. By adding up the counts of the gradient values, the gradient magnitude histogram can be made as well as the gradient direction histogram. The magnitude histogram is segmented to a $n_{1}$ dimensional vector while the direction histogram is segmented to a $n_{2}$ dimensional vector. Therefore, every patch becomes a $n_{1}+n_{2}$ dimensional vector. The training set consists of pre-labeled data points $\left(x_{i}, y_{i}\right)$, where $x_{i} \in R^{n_{1}+n_{2}}$ and $y_{i} \in\{+1,-1\}$ denotes it is a blurred image or a clear one.

The unknown image $I_{u}$, the same as the training data, is converted to one vector $x_{u}$ by the gradient magnitude histogram and the gradient direction histogram. The vector $x_{u} \in R^{n_{1}+n_{2}}$ will be classified by SVM and the output is one probability value $0 \leq p_{u} \leq 1$. If $p_{u}$ is higher, relatively, the chance $I_{u}$ belongs to a blurred image is higher.

\section{Blur Discrimination for Local and Global Blur}

In order to distinguish the locally blurred images with the globally blurred images, we segment the image into many grids and apply the blur estimation on each grid. A decision map is made to show where the blurred region is. The estimation does not need to be very precise since we just want to find locally or globally blurred image. Sometimes the monochrome scene with low gradients such as sky or wall might be treated as a blur region by the method described in the previous section. Thus, the monochrome identification is needed to eliminate the fake blur. Both monochrome region and blurred region have small gradient value, however, the difference between them is the color variance. By checking the variance with a threshold, we can verify if the region is blur or monochrome. After counting the number of blurred grids and non-blurred grids, the globally blurred image and locally blurred image can be discriminated.

\section{Classification of Globally Blurred Images}

\subsection{Point Spread Function}

Given a globally blurred image, it is possibly caused by either camera shake or out of focus. For a given blurred image $B$, the blurring effect can be modeled as $B=K \otimes L+N$, where $\otimes$ denotes the discrete image convolution and $L$ is the latent image convoluted by a blur kernel $K$ with sensor noise $N$. The blur kernel $K$ is a PSF, which is the key to distinguish the camera shake and out of focus, since they have significant difference. The PSF of a camera shake image has line structures and the blurred direction matches the PSF direction, but the PSF of an out of focus image is flat and dispersed, which means every pixel is polluted by nearby pixels. A common problem is that the blur kernel $K(x, y)$ is often unknown, or only partially known. In this case, the unknown $K$ has to be estimated from the blurred image $B$. 


\subsection{PSF Estimation}

Many PSF estimation algorithms have been proposed, and in this paper we follow the work of Fergus et al. [1]. The advantage of their algorithm is that only one image required. The approximation of the posterior distribution can be expressed by Bayes' Rule, and the variational algorithm minimizes a cost function that can represent the distance between the approximation and the true posterior. The parameters of the distributions are updated alternately by coordinate descent. The updates are repeated until the change converges. The mean of the distribution is then taken as the final value of the PSF.

\subsection{PSF Classification}

The PSF classification is rather similar to the blur estimation. The training images consist of half camera shake blurred images and half out of focus blurred ones. Every image is estimated to find the $n \times n$ blur kernel $K$, and the kernel is the feature of SVM classification. The kernel of every image is then transmuted, row by row, to a $n^{2}$ dimensional vector, and the model is built from these vectors. The predicting data, the same as training data, is converted to a vector from the estimated blur kernel, and then predicted to camera shake or out of focus by SVM.

\section{Classification of Locally Blurred Images}

If an image has some blurred regions and some clear regions at the same time, it is considered as a locally blurred image. There are two possible occasions that locally blurred region occurs: moving object and depth of field. To discriminate the two kinds of locally blurred images, we have to segment the foreground object from the background first. Image segmentation is a historical problem and there are many algorithms in this area. Pedro et al. [6] proposed an algorithm using a graph-based representation of the image and applied it to image segmentation. Their method is fast in practice and can preserve the details in low-variability regions while ignoring the details in high-variability regions.

Our goal is to find where the blurred region exactly is. For this reason, we apply the blur estimation on each region after the image segmentation to find the blur extent of each region. The fake blur problem arises here that the following two reasons may cause misclassification: 1 . if the region is too mall; 2 . if the region is monochrome. The fake blur can be judged by the neighboring pixels. If there is a region in the image with fake blur and its blur extent is unknown, we examine all pixels around the region to find the most frequent value and assign the value to the region.

In order to distinguish the moving object and depth of field, we apply the PSF estimation as described in the previous section. The estimated patch, however, is not decided only by the gradient. Since there are still some clear regions in the image, the PSF estimation should not be performed on the clear regions. We find 
the patch $P$ that can maximize the blur probability. The PSF classification on the patch can decide if the blurred patch is caused by motion or camera focus. If the blur is from motion, we can conclude that the image has moving object. If the blur is from camera focus, relatively, we can conclude that the image has depth of field.

\section{Results}

\subsection{Blur extent estimation}

From 200 blurred images and 200 clear images, half of the images are randomly chosen as the training data, and the remaining images are the predicting data. The gradient magnitude histogram is segmented to a 360 dimensional vector as well as the direction histogram. It turns out that the accuracy is $99.5 \%$ since only one image is wrong for 100 blurred images and 100 clear images. Fig. 3 shows some images and their blur extent values.
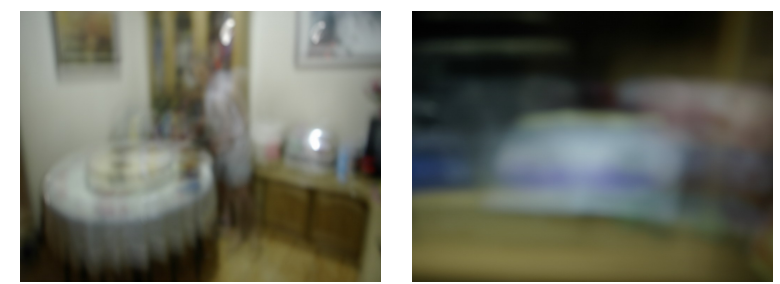

(a) Blur extent value $=($ b) Blur extent value $=$ 0.889063 0.879511
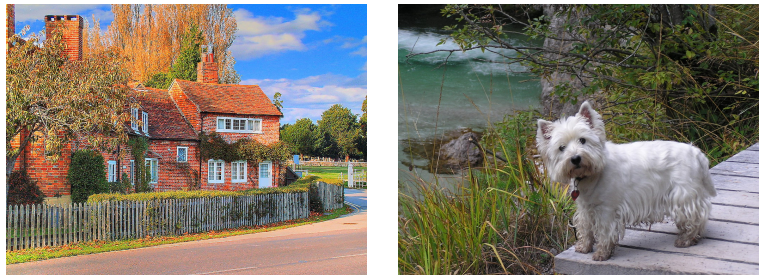

(c) Blur extent value $=(\mathrm{d})$ Blur extent value $=$ 0.007651 0.043577

Fig. 3. Different images and their blur extent values.

\subsection{Blur Discrimination for Local and Global Blur}

For those images with blur extent larger than 0.5, we segment the image into many grids to check if the blur is globally or locally. The width and the height of the grid may affect the prediction result: if the grids are too large, the prediction 
will not be accurate; if the grids are too small, there will be a multitude of fake blurred regions. The width and height of the grid is set to $10 \%$ of the image. If the prediction value of the grid is larger than 0.5 , it is a blurred region. The nonblurred region, vice versa, are the grids with value less than 0.5 . The variance of monochrome identification is heuristically set to 30. It turns out a decision map that can tell where the blurred region is. Every image is segmented to $k$ regions including $k_{1}$ blurred regions, $k_{2}$ non-blurred regions, and $k_{3}$ fake blurred regions in the decision map. If $k_{1} /\left(k_{1}+k_{2}\right) \geq 0.7$, then the image is a globally blurred image, otherwise it is a locally blurred image. Fig. 4 shows the grid structure and the visualization of the prediction result. The image is considered as a locally blurred image because there exist both blurred regions and non-blurred regions.
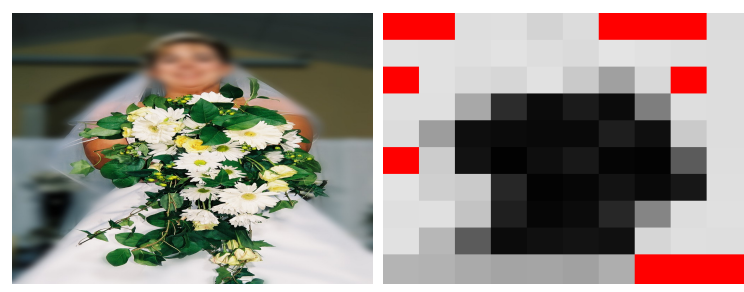

Fig. 4. Blur region detection.

\subsection{Classification of Globally Blurred Images}

From 80 camera shake blurred images and 80 out of focus image, half of the images are randomly chosen as the training data. We rescale all images to no more than $800 \times 600$ because the blur kernel size is related to the image size. Assuming the camera shake is not huge, we set the kernel size to $49 x 49$. Every image is then given a probability value $p, 0 \leq p \leq 1$ denotes the chance to be an out of focus image or a camera shake image. If $p$ is higher, relatively, the chance of the image to belongs to an out of focus image is higher. The prediction on the 40 camera shake images and 40 out of focus image results 4 wrong prediction which means the accuracy is $95 \%$. Fig. 5 shows the prediction on different globally blurred images and the estimated PSFs.

\subsection{Classification of Locally Blurred Images}

The accuracy of the classification of the locally blurred images is about $70 \%$ when predicting 40 depth of field and 40 moving object images. Fig. 6 shows the visualized result after segmentation, the blur estimation on each region with fake blur, and the final probability map. 

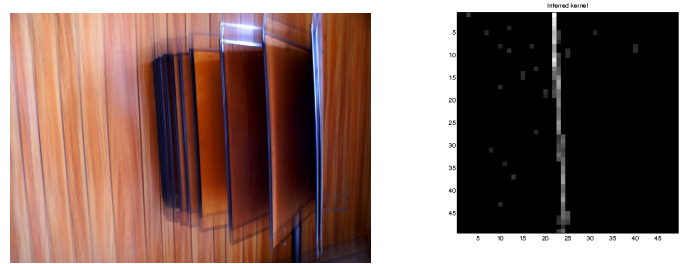

(a) PSF estimation probability $=0.038328$
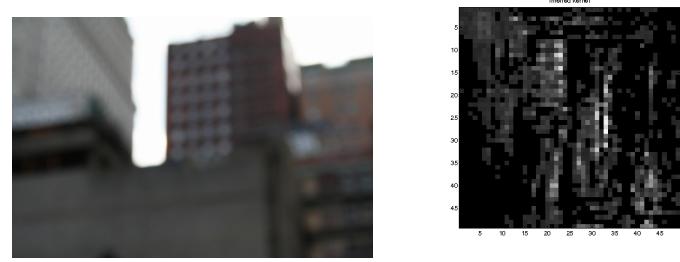

(b) PSF estimation probability $=0.842785$

Fig. 5. The classification of globally blurred images.

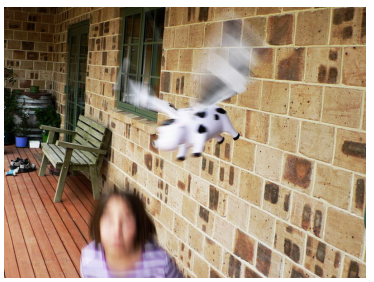

(a) Original image

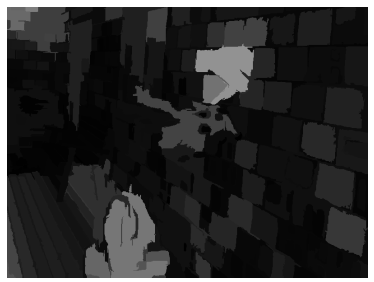

(b) Blur extent estimation (c) Estimated PSF on the result

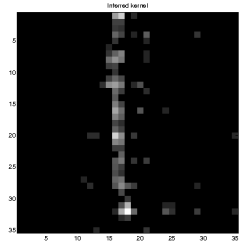

blurred region

Fig. 6. Locally blur detection.

\section{Conclusions and Future Work}

In this paper, we propose a framework to estimate the blur extent of an image and classify different types of blurred images. Blur extent estimation is performed on every input image, and those blurry image are chosen. The grid search is then applied to distinguish between locally blurred images and globally blurred ones. For the globally blurred image, we estimate its PSF and it can be classified into camera shake or out of focus. For the locally blurred image, we find the blurred regions using a segmentation method, and the PSF estimation on its blurred region can sort out the image with depth of field or moving object. The advantage of our framework is that the processes are automatic, so that the users can easily find the images they want by these hints.

The method proposed in [2] requires several rules and steps to calculate the blur extent of an image. Relatively, our method for blur extent estimation is highly accurate and not heuristic because the classification error is minimized 
automatically via SVM. Furthermore, our method can verify the images from different types of blur which is not proposed in other previous methods. The idea is new and has the potential for further researches.

\section{Acknowledgments}

This paper was partially supported by the National Science Council of Taiwan under NSC95-2622-E-002-018 and also by the Excellent Research Projects of the National Taiwan University under NTU95R0062-AE00-02.

\section{References}

1. Fergus, R., Singh, B., Hertzmann, A., Roweis, S.T., Freeman, W.T.: Removing camera shake from a single photograph. ACM Transactions on Graphics 25 (2006) 787-794 (SIGGRAPH 2006 Conference Proceedings).

2. Tong, H., Li, M., Zhang, H., Zhang, C.: Blur detection for digital images using wavelet transform. In: Proceedings of IEEE 2004 International Conference on Multimedia and Expo. (2004) 17-20

3. Elder, J.H., Zucker, S.W.: Local scale control for edge detection and blur estimation. In: Proceedings of 1996 European Conference on Computer Vision. Volume 2. (1996) 57-69

4. Ben-Ezra, M., Nayar, S.K.: Motion-based motion deblurring. IEEE Transactions on Pattern Analysis and Machine Intelligence 26(6) (2004) 689-698

5. Rooms, F., Philips, W., Portilla, J.: Parametric PSF estimation via sparseness maximization in the wavelet domain. In: Proceedings of SPIE Wavelet Applications in Industrial Processing II. (2004) 26-33

6. Felzenszwalb, P.F., Huttenlocher, D.P.: Efficient graph-based image segmentation. International Journal on Computer Vision 59(2) (2004) 167-181

7. Boser, B.E., Guyon, I.M., Vapnik, V.N.: A training algorithm for optimal margin classifiers. In: Proceedings of 1992 Workshop on Computational Learning Theory. (1992) 144-152

8. Cortes, C., Vapnik, V.: Support-vector networks. Machine Learning 20(3) (1995) 273-297

9. Platt, J.: Probabilistic outputs for support vector machines and comparison to regularize likelihood methods. In Smola, A., Bartlett, P., Schoelkopf, B., Schuurmans, D., eds.: Advances in Large Margin Classifiers. (2000) 61-74

10. Chang, C.C., Lin, C.J.: LIBSVM: a library for support vector machines. (2001)

11. Raskar, R., Agrawal, A., Tumblin, J.: Coded exposure photography: motion deblurring using fluttered shutter. ACM Transactions on Graphics 25 (2006) 795-804 (SIGGRAPH 2006 Conference Proceedings).

12. Miskin, J., MacKay, D.J.: Ensemble learning for blind image separation and deconvolution. In: Advances in Independent Component Analysis. (2000)

13. Reeves, S.J., Mersereau, R.M.: Blur identification by the method of generalized cross-validation. IEEE Transactions on Image Processing 1(3) (1992) 301-311 